\title{
Metallic Interaction in Polythiophene-Ti Plasma Composites
}

\author{
J. Cuauhtémoc Palacios ${ }^{1}$, Ma. Guadalupe Olayo ${ }^{2}$, Guillermo J. Cruz ${ }^{2 *}$ \\ ${ }^{1}$ Facultad de Ingeniería, Universidad Autónoma del Estado de México, Toluca, México \\ ${ }^{2}$ Departamento de Física, Instituto Nacional de Investigaciones Nucleares, Ocoyoacac, México \\ Email: "guillermoj.cruz@hotmail.com
}

Received March 24, 2013; revised April 24, 2013; accepted April 29, 2013

Copyright (C) 2013 J. Cuauhtémoc Palacios et al. This is an open access article distributed under the Creative Commons Attribution License, which permits unrestricted use, distribution, and reproduction in any medium, provided the original work is properly cited.

\begin{abstract}
This work presents a study about the structure and morphology of thin plasma composite films of polythiophene (PTh) with metallic particles of Ti (PTh-Ti). The objective was to study the water affinity and electric conductivity in PTh with the addition of metallic phases. The hydrophilicity was studied with the contact angles formed between water and surfaces, and the electric conductivity was calculated with the resistance measured in a two-probe device. The structure and morphology of the composites were studied with X-ray diffraction and scanning electron microscopy. The results indicated that PTh-Ti formed thin films of hybrid composites with oxidized Ti and Fe additional particles released from the electrodes used in the syntheses. The metallic fraction resulted in 2 diffraction angles at $31.6^{\circ}$ and $66.1^{\circ}$ product of the complex interaction among $\mathrm{Ti}$, resembling orthorhombic $\mathrm{TiO}_{2}$ structures, and $\mathrm{PTh}$. The metallic particles and iodine, added as a dopant to the composites to increase conductivity, reduced the hydrophilicity with water in a competition between roughness and chemical composition. In these conditions, the contact angles resulted between $46^{\circ}$ and $75^{\circ}$. The electric conductivity was about $10^{-6} \mathrm{~S} / \mathrm{m}$ increasing one order of magnitude with the metallic fraction. However, the high resistance of the polymeric fraction apparently dominates the transference of charges.
\end{abstract}

Keywords: Polythiophene; Titanium; Composites; Plasma

\section{Introduction}

Among polymeric materials, typically insulators, the group of conjugated heterocyclic polymers have relatively high electrical conductivity. This is the case of polythiophene (PTh), which has been used in solar cells and sensors since 1982, however its irregular efficiency led to a rapid decline in this application. Now, recent studies have focused on hybrid solar cells of PTh with materials with high electrical affinity, such as $\mathrm{TiO}_{2}$ [1-3]. The studies of ultrafast and efficient photo-induced electron transfer between conjugated polymers and fullerenes have represented an important step in the revival of these organic photovoltaic devices.

A hybrid solar cell is generally formed by at least four distinct layers: an anode, an electronic-hole transport layer, an active layer and a cathode. The active layer is responsible for light absorption and diffusion of charge carriers between donors and acceptors. In a cell of PTh, the anode would be made of indium-tin oxide (ITO); the

${ }^{*}$ Corresponding author. hole transport layer of a mixture of semiconductive polymers; the active layer would be formed of PTh with metallic particles; and the cathode would be a metallic conductor. Although it is known that the efficiency is higher with crystalline donors and acceptors [4], it has been found that non-crystalline semiconducting polymers and polymer-metal composites synthesized by plasma are very stable materials with promising electronic properties in these functions [5-7].

Considering these possibilities, the synthesis, structure, morphology and properties of plasma composites of PTh with $\mathrm{Ti}$ and I as active layers are studied in this work. Plasma techniques are direct methods free of solvents such as chloroform, chlorobenzene and tetrahydrofuran, commonly used in the synthesis of other hybrid solar cells based on semi-ordered polymers such as poly (3-hexyl) thiophene (P3HT). Many studies have focused on synthesizing this type of solar cells, eliminating solvents [8].

\section{Methodology}

The synthesis was performed in a cylindrical glass reac- 
tor of $9 \mathrm{~cm}$ diameter and $12 \mathrm{~cm}$ length. The base and work pressures were $5 \times 10^{-2}$ mbar and $7 \times 10^{-1} \mathrm{mbar}$, respectively. Two flat electrodes were used, the anode was made of stainless steel (SS) $6 \mathrm{~cm}$ diameter, and the cathode was made of $\mathrm{Ti}, 4 \mathrm{~cm}$ diameter, mounted on stainless steel bars. The separation between electrodes was $3 \mathrm{~mm}$.

PTh-Ti composites were synthesized in two steps using two power sources, $\mathrm{rf}$ and cd, switching during the synthesis. The first step was the synthesis of PTh with a Dressler rf power generator working at $13.56 \mathrm{MHz}$ and 20 W. During this step, a small amount of $\mathrm{Ti}$ was released from the electrodes. An Ar flow was used to moderate the flow of monomer into the reactor. To increase the metallic content, the second step was a Ti sputtering process performed with an Advanced Energy MDX magnetron drive at $1000 \mathrm{~V}$ with an Ar-air mixture as carrier gas. To achieve the Ti sputtering, the cathode was coupled with $0.127 \mathrm{~mm}$ thick Ti sharp foils. This allowed the sputtering of Ti and consequently, the synthesis of PThTi composites. To modify conductivity and hydrophilicity in the composites, PTh was synthesized adding I as a dopant in the first step, with the same metallic sputtering process in the second step. With this addition, PTh-I-Ti composites were formed.

The synthesis time was variable, for PTh, time intervals between 30 to $120 \mathrm{~min}$ were used, while for Ti sputtering the time intervals were between 15 to $40 \mathrm{~min}$. The composites were synthesized in the same chamber, being cautious that during polymerization the monomer feed rate was slow enough to avoid that the cathode was fully covered by the polymers. In the second step, the sputtering process was carried out alternating both $\mathrm{rf}$ and cd magnetron drives. Adjusting the monomer feed rate to the reactor, through a needle valve; the metal-polymer ratio in the composites was controlled. The composites were formed as thin films on glass substrates placed between the electrodes. In those substrates, the composites were analyzed.

The metallic structure was studied by X-ray diffraction with a Siemens D500 diffractometer from $2^{\circ}$ to $120^{\circ}$ in the typical $2 \theta$ scale. The metal fraction and its distribution in the polymer matrices were studied by electron microscopy and energy dispersive spectroscopy (EDS). The contact angles between composites and distilled water were obtained with drops of 8,12 and $16 \mu 1$ at $25^{\circ} \mathrm{C}$ in all cases. The dc electrical conductivity was evaluated by the two-point method at room temperature. It is important to note that this is a volumetric, not superficial, evaluation.

\section{Results and Discussion}

\subsection{Morphology of Composites}

The thickness of the films varied from approximately 1 to several micrometers, see Figure 1. During the synthesis, the monomer feed rate into the reactor had to be reduced in order to prevent that the Ti electrode become coated with the polymer, effect that would reduce the releasing of Ti particles. Consequently, as the metal quantity is increased, the composites are thinner than the polymeric layer without metal considering similar reaction time.

The morphology of PTh shows a homogeneously rough surface with several protrusions of about $1 \mu \mathrm{m}$ on layers of approximately $5 \mu \mathrm{m}$, see Figure 1(a). On the contrary, the surface of PTh-Ti composites looks smooth and homogeneous, see Figure 1(b). The addition of the metallic fraction reduced almost completely the protrusions on the surface, although the thickness of the metal layer cannot be differentiated from the substrate. However, there are zones in PTh-Ti where the surfaces were heavily attacked by the energetic plasma particles, near the electrodes, which appear with some pores and slight irregularities, see Figure 1(c).

The addition of I to PTh-Ti formed PTh-I-Ti composites in which many irregularities with particles and agglomerates randomly spread appeared on the surface, see Figure 1(d). The effect of I produced the release of $\mathrm{Fe}$ particles from the SS electrode, and this effect modified the smooth surface of PTh-Ti to roughened surfaces in PTh-I-Ti.

\subsection{Atomic Ratios}

The atomic ratios are tools used to analyze the participation of thiophene molecules, metals and dopants in the composites. Table 1 summarizes the elemental content of $\mathrm{C}, \mathrm{S}, \mathrm{I}, \mathrm{Ti}, \mathrm{O}$ and $\mathrm{Fe}$ in atomic percentage of all composite combinations. $\mathrm{C}$ and $\mathrm{S}$ are part of the thiophene structure (cyclic $-\mathrm{CH}=\mathrm{CH}-\mathrm{CH}=\mathrm{CH}-\mathrm{S}-$ ), but $\mathrm{O}$ is an additional oxidation in the polymers due to interaction with the atmospheric oxygen at the end of the synthesis. The participation of $\mathrm{Ti}$ is in the $0.34 \%-3.44 \%$ interval and $\mathrm{I}$ content is $13.89 \%$.

It is interesting to note that when iodine participates in the reaction $\mathrm{Fe}, 1.69 \%$, is released from the $\mathrm{SS}$ electrode. With the participation of iodine, the $\mathrm{Ti}$ content in the composites also increases, up to 10 times. This metal sputtering of $\mathrm{Ti}$ and $\mathrm{Fe}$ can be associated with collisions

Table 1. Elemental analysis of PTh, PTh-Ti and PTh-Ti-I composites in atomic percentage.

\begin{tabular}{cccccccc}
\hline Sample & $\mathbf{\% C}$ & $\mathbf{\% O}$ & $\mathbf{\% S}$ & $\mathbf{\% T i}$ & $\mathbf{\% F e}$ & $\mathbf{\% I}$ & $\mathbf{C / S}$ \\
\hline PTh & 80.35 & 6.83 & 12.82 & 0 & 0 & 0 & 6.27 \\
PTh-Ti & 79.32 & 5.81 & 14.53 & 0.34 & 0 & 0 & 5.46 \\
PTh-I-Ti & 46.26 & 10.12 & 24.60 & 3.44 & 1.69 & 13.89 & 1.88 \\
\hline
\end{tabular}




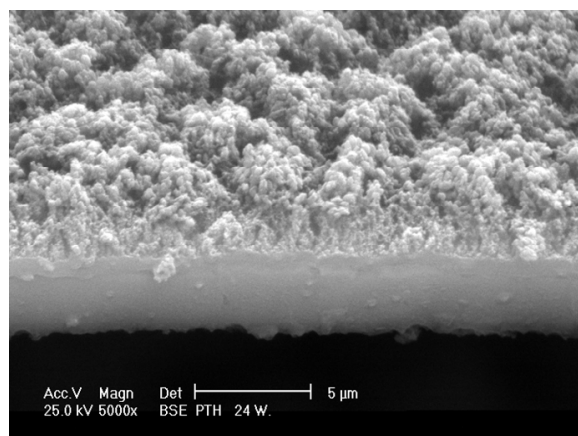

(a)

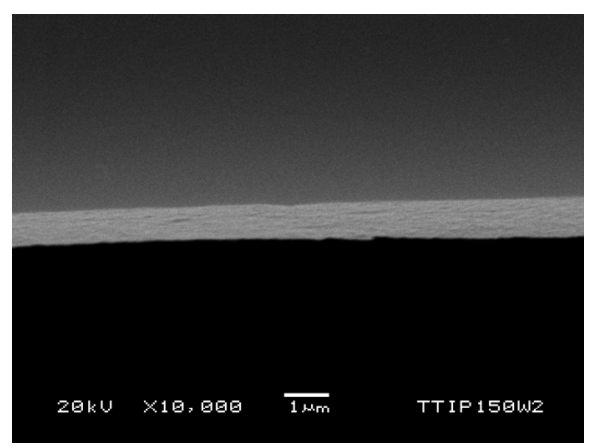

(b)

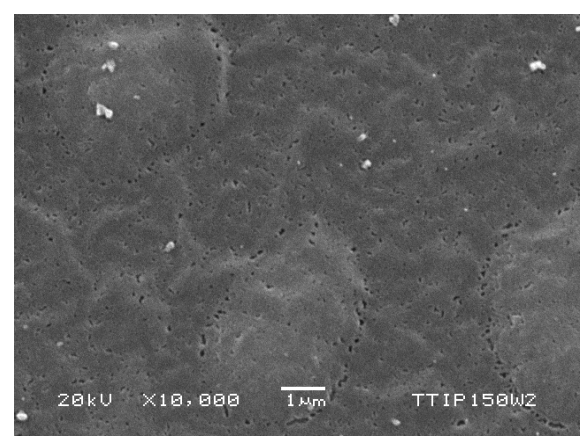

(c)

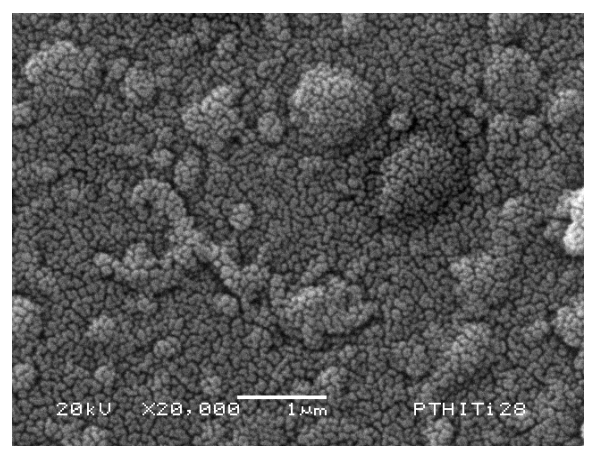

(d)

Figure 1. Morphology of: (a) PTh, rough surface with thickness of approximately $5 \mu \mathrm{m}$; (b) Lateral view of PTh-Ti, smooth surface with thickness of approximately $1 \mu \mathrm{m}$; (c) Surface of PTh-Ti showing the effect of the increased energy of plasma particles; and (d) Surface of PTh-I-Ti, rough surfaces could be associated with the inclusion of iodine in the synthesis. (a) PTh. (b) PTh-Ti. (c) PTh-Ti Surface. (d) PTh-I-Ti Surface. with I ions in the plasma. The $\mathrm{O}$ content also reflects the iodine influence increasing the $\mathrm{O}$ atomic content, from $5.81 \%$ in PTh-Ti to $10.12 \%$ in PTh-I-Ti. In PTh-Ti is observed the lowest $\mathrm{O}$ content. Another origin of $\mathrm{O}$ in the composites could be in the oxygen atoms participating in both electrodes.

The increase of metal and oxygen percentages reduces the $\mathrm{C}$ participation on the surface with iodine. In PTh and PTh-Ti, the $\mathrm{C}$ content is approximately $80 \%$, but reduces to $46.26 \%$ as a consequence of iodine. The $\mathrm{C} / \mathrm{S}$ atomic ratio is 4 in thiophene and it would be approximately the same in polythiophene composites without molecular fragmentation. However, the $\mathrm{C} / \mathrm{S}$ ratio obtained was different from 4 , in $\mathrm{PTh} \mathrm{C} / \mathrm{S}=6.27$ indicating molecular fragmentation associated with the high energy of plasma particles in which some sulfur fragments did not participate in the composite, probably because they escaped from the reactor. On its part, PTh-Ti has a moderate amount of metal, with $\mathrm{C} / \mathrm{S}$ smaller than $\mathrm{PTh}, 5.46$, but remains high relative to $\mathrm{C} / \mathrm{S}$ in thiophene. This ratio indicates smaller fragmentation than the case before. The lowest $\mathrm{C} / \mathrm{S}$ ratio was found in PTh-I-Ti, 1.88, suggesting that the more metal in the composites the smaller the $\mathrm{C} / \mathrm{S}$ ratio. This ratio also indicates fragmentation, but in a different way than the previous cases in which what escaped from the reactor were the carbon fragments and not the sulfur ones because of the iodine participation. It is clear that the high energy associated with the sputtering is able to fragment thiophene rings with the consequence that some of the smaller molecules are extracted from the reactor by the vacuum system.

\subsection{Contact Angles}

The physical and chemical composition of surfaces plays a key role in the hydrophilic behavior of materials. The forces on the surface can be modified with the addition of superficial layers of different materials. In PTh-Ti, the surface tension increases with the metallic fraction and small contact angles are observed, see Figure 2, in which regardless the synthesis conditions, the films show hydrophilic behavior with angles below $90^{\circ}$.

Contact angles of all PTh composites with distilled water were calculated between $46^{\circ}$ and $75^{\circ}$. PTh has the greatest values, $72^{\circ}-75^{\circ}$, associated with its rough surface. The metallic particles added to PTh greatly reduced the roughness on the surface and as a consequence, the contact angles in PTh-Ti reduced to the $62^{\circ}-70^{\circ}$ interval. The dopant, iodine, participating in PTh-I-Ti increased the roughness on the surface with respect to PTh-Ti but unexpectedly further reduced the angles sending them to the $46^{\circ}-55^{\circ}$ interval. This effect could be due to the hygroscopic character of I atoms inserted on the surface, which appears to have more influence than the hydro- 
phobicity associated with the superficial roughness, see Figure 1(d).

It is worthy to note this effect, because PTh-I-Ti is an example of the great influence of chemical composition of surfaces, because although its surface is much rougher, the inclusion of iodine reduced the contact angles to the lowest values, making it the most hydrophilic composite.

\subsection{Diffraction Angles}

The X-ray diffraction of PTh-I-Ti composites is shown in Figure 3 and presents superposition of both phases, metallic and polymeric. Figure 3 also includes the main diffraction lines of $\mathrm{TiO}_{2}$ and $\mathrm{Fe}_{2} \mathrm{O}_{3}$ oxides to compare with those of the composites, because, as it was shown in

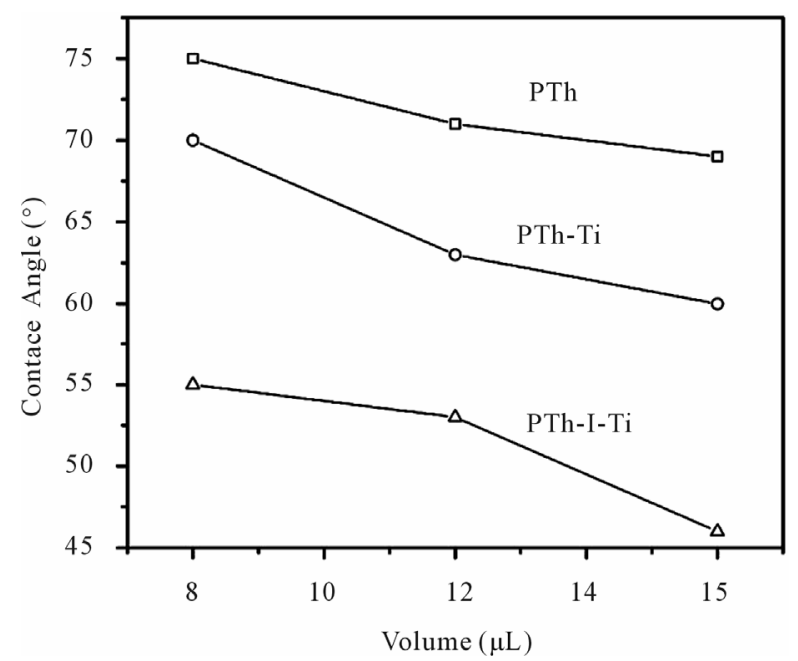

Figure 2. Contact angles of PTh composites. They are the result of their physicochemical characteristics, roughness and chemical composition of surfaces.

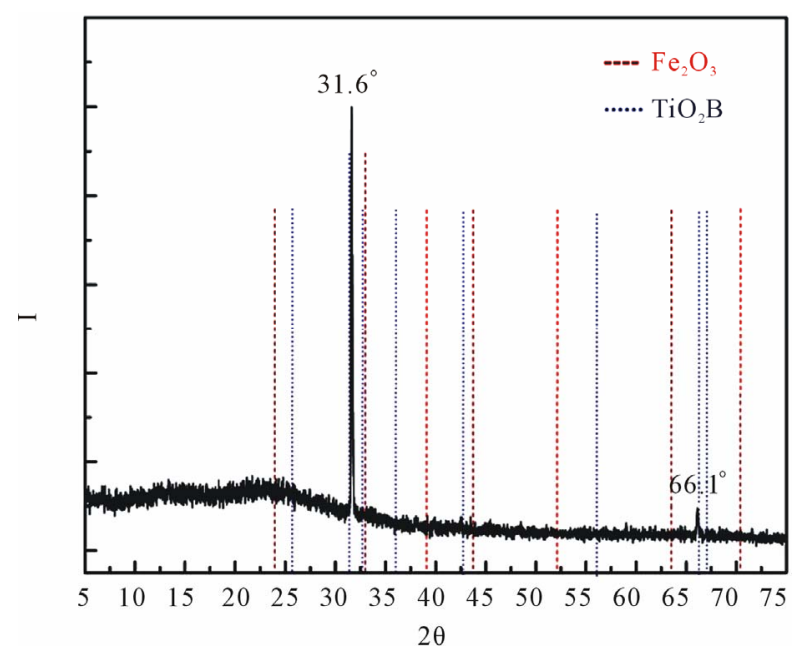

Figure 3. X-ray diffraction pattern of PTh-I-Ti. Well defined peaks at $31.6^{\circ}$ and $66.1^{\circ}$ can be observed. Dashed (in red) and dotted (in blue) lines represent the diffraction angles of $\mathrm{Fe}_{2} \mathrm{O}_{3}$ and $\mathrm{TiO}_{2}$ (Brookite), respectively.
Table 1, PTh-I-Ti has $3.44 \%$ of Ti and $1.69 \%$ of Fe. The diffractogram shows the prevalence of the polymeric amorphous phase upon the metal, although there are two well defined signals protruding from the amorphous level at $31.6^{\circ}$ and $66.1^{\circ}$ that can be associated with two diffraction angles of the $\mathrm{TiO}_{2}$ orthorhombic brookite structure $[9,10]$. This structure has been reported with photocatalytic activity which can be useful in the properties of PTh-Ti composites of this work. $\mathrm{Fe}_{2} \mathrm{O}_{3}$, on its part, do not diffract in those angles, however, the one at $24^{\circ}$ is overlapped with the amorphous centroid of PTh.

These results suggest that $\mathrm{Ti}$ and $\mathrm{Fe}$ atoms arranged in a complex interaction with the polythiophene substrate. Considering that both metals start the interaction with PTh without previous combinations with $\mathrm{C}$, the diffraction suggests that they form structures with $\mathrm{C}$ and $\mathrm{O}$ in contact with PTh. Periodic structures of Ti surrounded by $\mathrm{O}(\mathrm{O}-\mathrm{Ti}-\mathrm{O})$ planes are not discarded.

\subsection{Electric Conductivity}

The volumetric electric conductivity of PTh composites calculated at room temperature is summarized in Table 2. As shown, conductivity in PTh is $3.52 \times 10^{-6} \mathrm{~S} / \mathrm{m}$ and increased in PTh-Ti one order of magnitude with the metal content, $20.6 \times 10^{-6} \mathrm{~S} / \mathrm{cm}$. However, with the inclusion of I, conductivity in PTh-I-Ti was reduced even to lower values than those of PTh, $1.8 \times 10^{-6} \mathrm{~S} / \mathrm{m}$. This reduction can be the result of a competitive effect between I and Ti on the surface, in which both look to have opposite influence on the transference of electric charges.

The influence of metals in polymers is great in the transference of charges in volumetric polymer-metal matrices; however metals in layered polymer-metal composites do not have the same effect, because the main resistance of polymeric layers dominates the transference. In these conditions, the level of conductivity in PTh-Ti composites is between semiconductors and non-conductors, because the constant impact of energetic particles promotes branching and crosslinking in the polymeric phase reducing the movement of electric charges across PTh matrices. However, polymeric films for hybrid solar cells need photoelectric effects and a good union between polymers and metals so the charges can flow to the electrodes of the cell. This kind of union is accomplished in PTh-Ti composites.

Table 2. Electrical conductivity of PTh composites at room temperature.

\begin{tabular}{cc}
\hline Sample & $\sigma(\mathrm{S} / \mathrm{m})$ \\
\hline PTh & $3.52 \times 10^{-6}$ \\
PTh-Ti & $20.6 \times 10^{-6}$ \\
PTh-I-Ti & $1.8 \times 10^{-6}$ \\
\hline
\end{tabular}




\section{Conclusions}

Plasma PTh-Ti layered composites were synthesized by plasma to study the morphology, hydrophilicity and transference of electric charges. The syntheses were combinations of polymerization, doping and sputtering of metals. The results indicated that the high energy associated with the sputtering has the disadvantage of breaking some thiophene rings, but on the other hand it has the advantage of joining polymers and metals in hybrid composites. The metallic fraction was composed by $\mathrm{Ti}$ and Fe partially combined with the polymeric fraction giving 2 diffraction angles at $31.6^{\circ}$ and $66.1^{\circ}$ which can be associated with two diffraction angles of orthorhombic $\mathrm{TiO}_{2}$ brookite. The amorphous diffraction PTh spectrum looks overlapped with structures of $\mathrm{Ti}$ and possibly Fe. The metallic particles released from the electrodes may combine with the substrate in complex arrangements at the interface.

All the composites showed hydrophilic behavior with contact angles between distilled water and composites from $46.1^{\circ}$ to $74.6^{\circ}$. Despite the roughness, the chemical composition of surfaces played a key role in the hydrophilic behavior of materials in which the participation of iodine increased the roughness on the surface but reduced the contact angles as a consequence of its affinity with water. The transference of charges increased one order of magnitude in the composites with the metal inclusion, but reduced approximately the same magnitude with the participation of iodine atoms, both components produced opposite effects.

\section{Acknowledgements}

The authors acknowledge CONACYT in Mexico for the partial financial support to this work with the projects 154757 and 130190. J.C. Palacios acknowledges the financial support of PROMEP-MEXICO with the project FE032/2012-351030.

\section{REFERENCES}

[1] Q. Qiao and J. McLeskey, "Water-Soluble Polythiophene/ Nanocrystalline $\mathrm{TiO}_{2}$ Solar Cells," Applied Physics Letters, Vol. 86, No. 12, 2005, Article ID: 153501. doi:10.1063/1.1900300
[2] N. Gerein, M. Fleischauer and M. Brett, "Effect of $\mathrm{TiO}_{2}$ Film Porosity and Thermal Processing on $\mathrm{TiO}_{2}-\mathrm{P} 3 \mathrm{HT}$ Hybrid Materials and Photovoltaic Device Performance," Solar Energy Materials and Solar Cells, Vol. 94, No. 12, 2010, pp. 2343-2350.

[3] B. Reeja-Jayan and A. Manthiram, "Influence of PolymerMetal Interface on the Photovoltaic Properties and LongTerm Stability of nc-TiO 2 -P3HT Hybrid Solar Cells," Solar Energy Materials and Solar Cells, Vol. 94, No. 5, 2010, pp. 907-914.

[4] J. Chandrasekaran, D. Nithyaprakash, S. Maruthamuthu, D. Manoharan and S. Kumar, "Hybrid Solar Cell Based on Blending of Organic and Inorganic Materials-An Overview," Renewable and Sustainable Energy Reviews, Vol. 15, No. 2, 2011, pp. 1228-1238. doi:10.1016/j.rser.2010.09.017

[5] J. C. Palacios, G. J. Cruz, M. G. Olayo and J. ChávezCarvayar, "Characterization of Hydrophobic and Hydrophilic Polythiophene-Silver-Copper Thin Film Composites Synthesized by DC Glow Discharges," Surface and Coatings Technology, Vol. 203, No. 20-21, 2009, pp. 3032-3036. doi:10.1016/j.surfcoat.2009.03.020

[6] G. J. Cruz, J. C. Palacios, M. G. Olayo, J. Morales and R. Olayo, "Conductivity of Layer and Matrix PolyanilineSilver-Copper Composites by Plasma," Journal of Applied Polymer Science, Vol. 93, No. 3, 2004, pp. 10311036. doi:10.1002/app.20545

[7] J. C. Palacios, M. G. Olayo, J. Morales, G. J. Cruz and R. Olayo, "Plasma Formation of Polymer-Metal Composites," International Journal of Polymeric Materials, Vol. 51, No. 6, 2002, pp. 529-536.

[8] G. Thalluri, J.-C. Bolsée, A. Gadisa, M. Parchine, T. Boonen, J. D'Haen, A. Boyukbayram, J. Vanderbergh, T. Cleij, L. Lutsen, D. Vanderzande and J. Manca, "Opto-Electrical and Morphological Characterization of Water Soluble Conjugated Polymers for Eco-Friendly Hybrid Solar Cells," Solar Energy Materials and Solar Cells, Vol. 95, No. 12, 2011, pp. 3262-3268.

[9] A. Di Paola, M. Addamo, M. Bellardita, E. Cazzanelli and L. Palmisano, "Preparation of Photocatalytic Brookite Thin Films," Thin Solid Films, Vol. 515, No. 7-8, 2007, pp. 3527-3529. doi:10.1016/j.tsf.2006.10.114

[10] M. P. Moret, R. Zallen, D. P. Vijay and S. B. Desu, "Brookite-Rich Titania Films Made by Pulsed Laser Deposition," Thin Solid Films, Vol. 366, No. 1-2, 2000, pp. 8-10. doi:10.1016/S0040-6090(00)00862-2 